
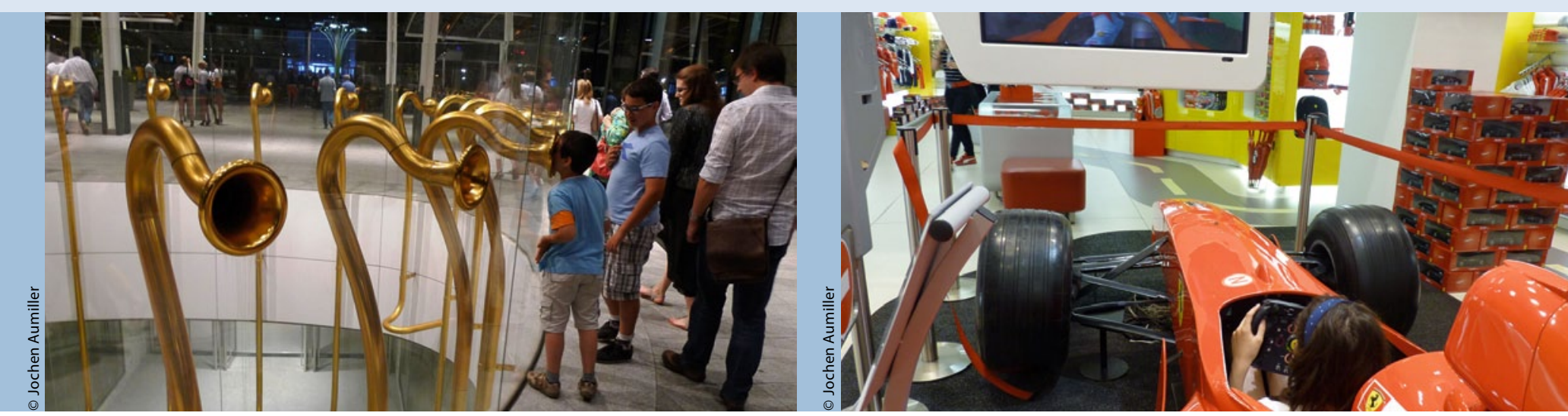

schätzung des absoluten Mortalitätsrisikos für die nächsten zehn Jahre liefert. Wird aufgrund eines niedrigen Risikos nur unzureichend behandelt, kann es nach Jahren zu einer irreversiblen Hochrisiko-Situation kommen. So wird geraten, bei jungen Personen das relative Risiko oder das Gefäßalter abzuschätzen. Entsprechende Charts bietet die „Joint European Societies' Guidelines on CVD Prevention in Clinical Practice“.

\section{Selbstmessung wird aufgewertet}

Bislang galt die Druckmessung in der Arztpraxis als goldener Standard der Blutdruckdiagnostik. Auch in zahlreichen Studien wurde diese verwendet. In den neuen Leitlinien wird der Stellenwert der außerhalb der Praxis erhobenen Druckwerte betont, entweder mit der ambulanten 24-Stunden-Messung (ABPM) oder mithilfe der Selbstmessung zuhause (HBPM). Gerade die

\title{
Offene Fragen, schwache Evidenzen
}

Obwohl die neuen Leitlinien 735 Literaturstellen auflisten, bedeutet das noch lange nicht, dass alle Empfehlungen durch überzeugende Evidenzen getragen werden. Darauf legen auch die Autoren der Leitlinien großen Wert. Am Ende des Artikels zählen sie zahlreiche Wissenslücken auf, die aktuellen Forschungsbedarf markieren. Hier eine Auswahl von dringenden offenen Fragen:

_ Sollte die antihypertensive Pharmakotherapie schon für alle Patienten mit einer Hypertonie vom Grad 1 vorgesehen werden, auch wenn ihr kardiovaskuläres Risikoprofil niedrig bis mäßig ist?

_Bei alten Hypertonikern: Sollte ein systolischer Druck zwischen 140 und $160 \mathrm{mmHg}$ wirklich mit Antihypertensiva behandelt werden?

— Weißkittelhypertonie: Ist die Therapie dieser Hypertonieform mit Antihypertensiva sinnvoll oder wäre es nicht besser, einen Weg zu suchen, Patienten, die keine Therapie benötigen, von solchen zu differenzieren, die behandelt werden sollten?

_ Ist die antihypertensive Pharmakotherapie bereits bei Personen mit hoch normalen Druckwerten (130-139/85-89 mmHg) angezeigt?

- Was sind die optimalen Druckwerte bei der Praxismessung (d. h. die protektivsten und sichersten), wie sie in unterschiedlichen demografischen und klinischen Situationen erreicht werden sollten?

_ Bieten Behandlungskonzepte, die auf der ambulanten Messung und Selbstmessung beruhen, Vorteile gegenüber der Praxismessung (niedrigere Morbidität und Mortalität? Weniger Pharmaka und Nebenwirkungen?).

_ Welche optimalen Druckwerte sollen bei der ambulanten Messung und Selbstmessung zuhause angestrebt werden, und sollten die Zielwerte für Hochrisikopatienten niedriger oder höher sein?

_ Bringt der zentrale Blutdruck Zusatzinformationen für die Vorhersage von kardiovaskulären Ereignissen sowohl bei behandelten wie auch bei unbehandelten Personen?

_ Schneiden interventionelle Verfahren zur Therapie der resistenten Hypertonie günstig ab im Vergleich zur bestmöglichen Pharmakotherapie? Werden dadurch auch eine langfristige Druckkontrolle und eine Reduktion von Ereignissen erreicht?

_ Lebensstilveränderungen: Bedeutet die Erniedrigung des Blutdrucks tatsächlich eine Reduktion von Morbidität und Mortalität?

HBPM bietet wegen der häufigen Verlaufsmessungen viele Vorteile. Und es ist zu erwarten, dass über die Verwendung von speziellen Smartphone-Applikationen oder via Telemonitoring der Aussagewert der HBPM noch gesteigert wird.

Vor allem kann mit der HBPM der sogenannte Weißkittelhochdruck aufgedeckt werden, also jene Druckabweichung, die lediglich in der Arztpraxis gemessen wird, nicht jedoch zuhause. Bevölkerungsstudien ergaben, dass die Weißkittelhypertonie durchschnittlich 13\% der Hypertonieprävalenz ausmacht. Ein nicht zu unterschätzendes Praxisproblem also, das zu Fehl- bzw. Übertherapie geradezu einlädt.

Ferner schlägt mengenmäßig auch die maskierte Hypertonie zu Buche. Hierbei wird in der Praxis ein hochnormaler Druck gemessen, außerhalb jedoch ein erhöhter. Dann droht vor allem eine Untertherapie, wenn der Praxiswert als einziger erhoben wird.

In der Leitlinien von 2007 war die Evidenz für die 24-Stunden-Messung nachhaltig betont worden, nicht jedoch die der Selbstmessung. Das hat sich geändert. Nicht zuletzt auch wegen der prognostischen Bedeutung der HBPM, ihrer simplen Durchführbarkeit sowie ihrer günstigen Kosten-Nutzen-Relation.

Nach neueren Metaanalysen korreliert die HBPM enger mit Organschäden, speziell mit der linksventrikulären Hypertrophie, als die Praxismessung. Aber auch der Vorhersagewert für kardiovaskuläre Ereignisse ist besser. ABPM und HBPM sollte man nicht als konkurrierende, sondern vielmehr als komplementäre Verfahren ansehen, denn sie liefern unterschiedliche Informationen.

Ein Minuspunkt für die Heimmessung im Vergleich zum ABPM ist das Fehlen nächtlicher Druckwerte. Diese haben sich jedoch prognostisch als we- 